\title{
Laboratory Nanoparticle X-Ray Fluorescence Tomography for High-Resolution Small-Animal Imaging
}

\author{
$\underline{\text { Jakob C. Larsson }}^{1,}{ }^{*}$, Carmen Vogt $^{1}$, William Vågberg ${ }^{1}$, Muhammet S. Toprak ${ }^{1}$, Johanna Dzieran ${ }^{2}$, Marie \\ Arsenian-Henriksson ${ }^{2}$, and Hans M. Hertz ${ }^{1}$ \\ 1. Department of Applied Physics, KTH Royal Institute of Technology/AlbaNova, 10691 Stockholm, \\ Sweden. \\ 2. Department of Microbiology, Tumor and Cell Biology (MTC), Karolinska Institutet, 17177 \\ Stockholm, Sweden. \\ * Corresponding author, jakob.larsson@biox.kth.se
}

Laboratory nanoparticle x-ray fluorescence tomography is an emerging imaging technique with potential for high-spatial-resolution molecular small-animal imaging [1-3]. In this technique the $\mathrm{x}$-ray fluorescence (XRF) signal from targeted metal-core nanoparticles (NPs) is measured, providing information about both the spatial distribution and concentration of the NPs inside the object. However, present laboratory XRF tomography systems typically have limited spatial resolution $(>1 \mathrm{~mm})$ and suffer from long scan times and high radiation dose even at high NP concentrations, mainly due to low efficiency and poor signal-to-noise ratio (SNR). Other macroscopic bioimaging methods offers either high spatial resolution without molecular contrast (e.g., x-rays) or molecular contrast with lower resolution (e.g., PET, SPECT). Synchrotrons frequently use XRF for NP imaging but the lowdivergence beam and limited access to these facilities makes them less applicable for small-animal imaging [4]. We have developed a laboratory XRF tomography system using Mo NPs that provides high spatial resolution (sub-200 $\mu \mathrm{m}$ ), high sensitivity and vastly decreased scan times and dose, opening up the possibilities for in-vivo small-animal imaging research [5].

Figure 1 depicts the experimental arrangement. The system consists of a high-brightness liquid-metal-jet microfocus x-ray source (D2, Excillum AB, Sweden), multicoated Montel x-ray focusing optics (ELM61, Incoatec GmbH, Germany) and two photon counting detectors (X123, Amptek Inc., MA). The source operates at $170 \mathrm{~W} / 120 \mathrm{kVp}$ and emits a polychromatic spectrum with strong In $\mathrm{K} \alpha$ line emission at $24.1 \mathrm{keV}$. This line is selected by the Montel mirror's W-C-coating and focused into a semimonochromatic pencil beam, creating a $\sim 100 \times 100 \mu \mathrm{m}$ focus $56 \mathrm{~cm}$ from the source. The brilliance in the focus was measured to $8 \times 10^{8} \mathrm{ph} /\left(\mathrm{s} \times \mathrm{mrad}^{2} \times \mathrm{mm}^{2} \times\right.$ line $)$. The Mo NPs are intravenously injected into the tail vein of the mouse at a predetermined time before the scan. Mo has its $\mathrm{K}$ absorption edge at $20.0 \mathrm{keV}$ which nicely matches the pencil beam energy. Upon excitation by the pencil beam the NPs emit $\mathrm{K} \alpha$ fluorescence at $17.4 \mathrm{keV}$. This energy matching of the source, optics and NPs greatly reduces the Compton background, increasing the SNR and enabling short scan times $(25 \mathrm{~ms} /$ pixel) and low radiation dose $(<50 \mathrm{mSv})$. Each measurement provides information about both the spatial distribution and concentration of the Mo nanoparticles, as well as the x-ray absorption of the object (regular CT image). The data is acquired using an in-house built "on-the-fly" scan software. The mouse is scanned continuously in the horizontal direction but the acquisition is paused during vertical and rotational movements. A regular filtered back-projection algorithm is used to reconstruct the CT image while an iterative reconstruction method is used to reconstruct the XRF image.

Using this system, a series of XRF measurements were performed on mice. At a predetermined time (15 min -16 weeks) before the measurement, $100 \mu \mathrm{l}$ of $1.5 \mathrm{wt} \%$ Mo NPs solution was injected into the tail 
vein of the mouse. The mouse was then sacrificed and scanned. The acquisition time was $50 \mathrm{~ms} / \mathrm{step}$ and step size $200 \mu \mathrm{m}$ in both the horizontal and vertical directions. Figure 2 shows orthogonal projection images of the NP bio-distribution in four mice at different times after the injection. The pixel size is $200 \times 200 \mu \mathrm{m}^{2}$. The absorption image is in black/white and the XRF signal is in color. The percentage in the title indicate how much of the NPs are left in the body compared to after 15 min. After 15 min the NPs are spread out to all major organs, including the brain, lungs, liver and spleen. As times passes the NPs are caught by macrophages in the liver and slowly excreted. After 4 weeks there's no NPs left in the lungs and spleen, and after 16 weeks only $\sim 1 \%$ of the injected NPs are still left in the body.

\section{References:}

[1] M. Bazalova et al, IEEE Trans. Med. Imaging 31 (2012) 1620.

[2] H. M. Hertz et al, Opt. Lett. 39 (2014) 2790.

[3] N. Manohar et al, Sci. Rep. 6 (2016) 22079.

[4] See, e.g., T. Paunesku et al, Nat. Materials 2 (2003) 343.

[5] J. C. Larsson et al, Submitted.

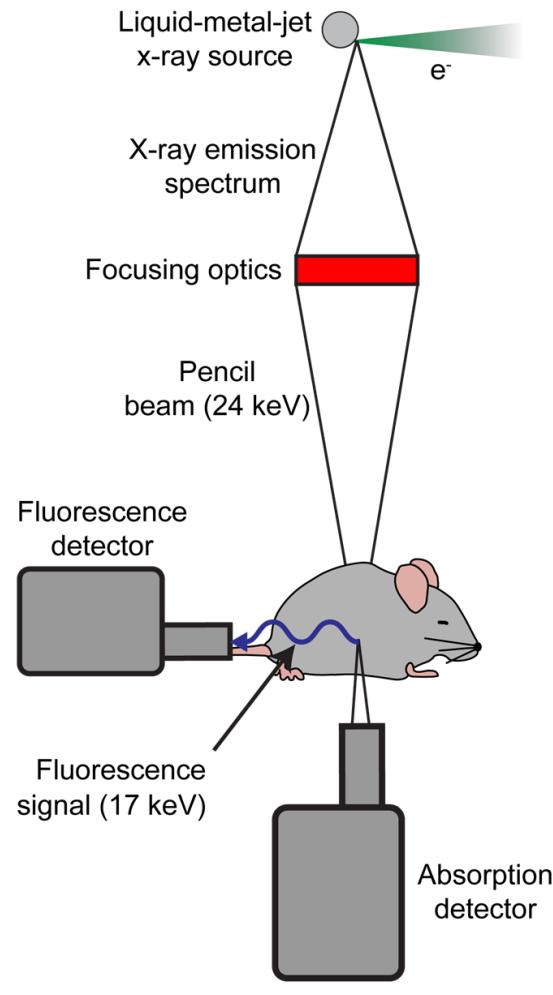

Figure 1. Experimental arrangement. A semi-monochromatic pencil beam is used to probe the spatial distribution and concentration of Mo NPs in the mouse.

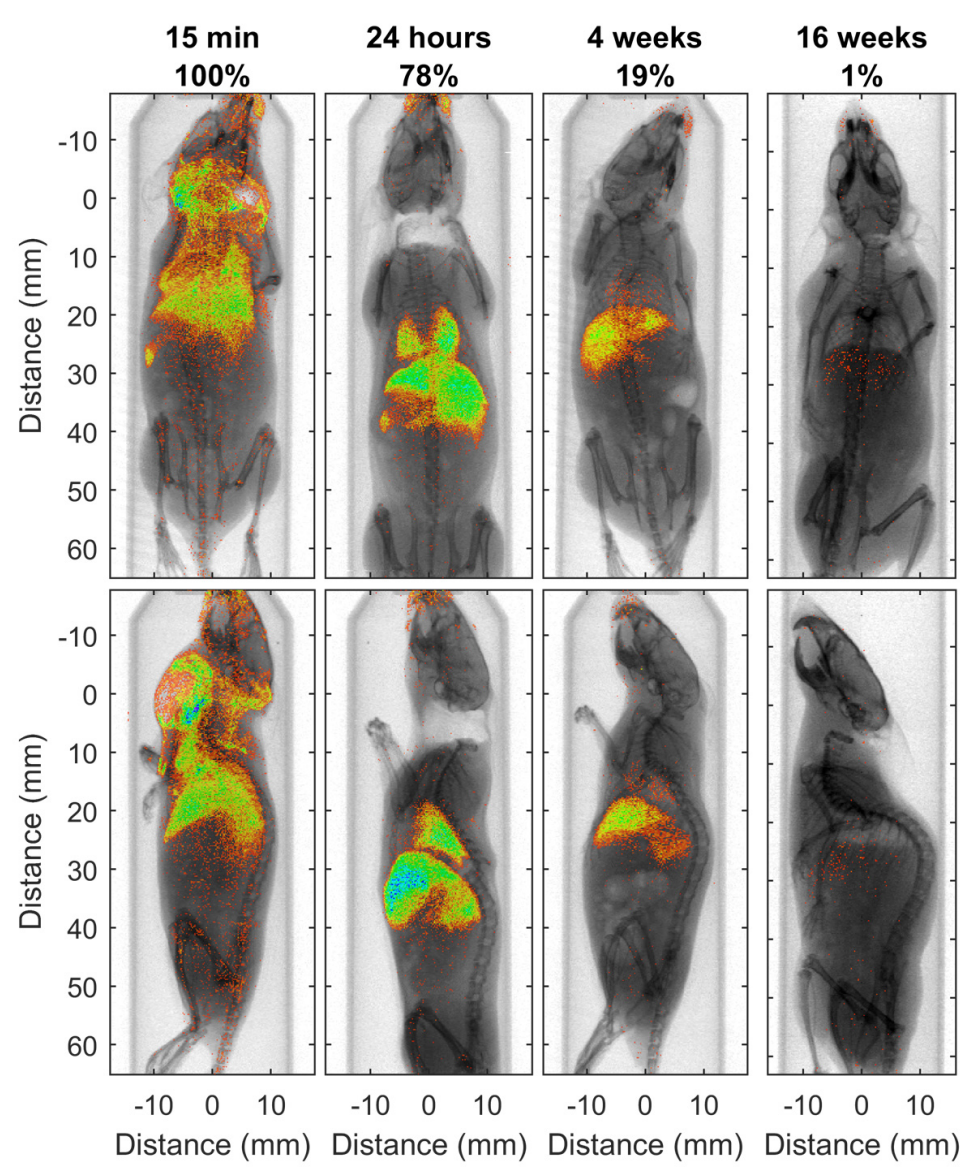

Figure 2. Overlay projection images of the nanoparticle biodistribution at different times after the injection. CT image in black and white and XFCT image in colors. The percentage in the title indicates how much of the nanoparticles that are left in the body compared to after $15 \mathrm{~min}$. 